\title{
ARTRODIASTASE PARA O TRATAMENTO DA RIGIDEZ DO QUADRIL NA ARTRITE REUMATOIDE JUVENIL (ARJ) - RESULTADOS PRELIMINARES
}

\author{
ARTHRODIASTASIS FOR THE TREATMENT OF STIFFNESS OF THE HIP IN JUVENILE \\ RHEUMATOID ARTHRITIS (JRA) - PRELIMINARY RESULTS
}

Roberto Guarniero', Rui Maciel de Godoy Júnior ${ }^{2}$, Nei Botter Montenegro3, Carlos Augusto Malheiros Luzo ${ }^{3}$, Maria Helena Bittencourt Kiss ${ }^{4}$

\section{RESUMO}

Objetivo: Apresentar os resultados preliminares da utilização da artrodiastase do quadril em pacientes portadores de artrite reumatoide juvenil e com comprometimento da articulação coxofemoral. Métodos: Estudo prospectivo de 12 pacientes (seis meninos e seis meninas) com idades entre oito e 18 anos (média de 10,5 anos). Foi utilizado um fixador externo monolateral que permite os movimentos de flexão e de extensão no quadril. O fixador externo foi mantido por um período que variou de 78 a 90 dias, com média de 86 dias. O controle radiográfico foi realizado durante $\mathrm{o}$ ato operatório e, semanalmente, durante o período de tração e a cada quatro semanas, quando terminado este período. Na avaliação clínica dos resultados, incluímos a graduação da dor e o grau de movimentação articular, com medidas e avaliações pré e pós-operatórias. O período de acompanhamento variou de 12 a 15 anos, com média de 13 anos. Resultados: O valor médio da escala de dor foi de nove (9) antes da operação e de quatro (4) no período pós-operatório. Em dois pacientes não ocorreu melhora da dor. O arco de movimento do quadril aumentou em todos os pacientes, com exceção de dois. $\mathrm{Na}$ avaliação radiográfica evidenciamos um aumento no espaço articular de $2 \mathrm{~mm}$, em média, e que se manteve no pós-operatório. Não foram observadas complicações com a utilização da técnica. Apenas verificamos soltura dos pinos de Schanz da região do osso ilíaco em dois pacientes. A técnica operatória não ocasionou resultado satisfatório. Conclusão: O procedimento de artrodiastase está bem indicado para a recuperação da mobilidade em uma articulação coxofemoral comprometida e rígida, como ocorre em pacientes com artrite reumatoide juvenil.

Descritores - Quadril; Artrite Reumatoide Juvenil; Procedimentos Ortopédicos

\section{ABSTRACT}

Objective: To present the preliminary results of the use of hip arthrodiastasis in patients with juvenile rheumatoid arthritis and involvement of the hip joint. Methods: A prospective study of 12 patients (six boys and six girls) aged between eight and 18 years (mean 10.5 years). We used a monolateral external fixator that allows flexion and extension at the hip. The external fixator was maintained for a period ranging from 78 to 90 days, with a mean of 86 days. Radiographic control was performed during surgery, weekly during the traction period, and every four weeks once this period was completed. The clinical evaluation of results included the degree of the pain and the degree of joint movement, measured and evaluatedpreand post-operatively. The follow-up period ranged from 12 to 15 years, with a mean of 13 years. Results: The average pain score was nine (9) before surgery and four (4) in the postoperative period. There was no improvement in pain in two patients. The range of motion of the hip increased in all patients except two. Radiographic evaluation evidenced a2 mm increase in joint space, on average, that has remained postoperatively. There were no complications with this technique. Only a loosening of the Schanz screws in the region of the iliac bone was observed in two patients. The surgical technique did not bring satisfactory results. Conclusion: The arthrodiastasis procedure is well suited for recovery of mobility in animpairedand rigid hip joint, as occurs in patients with juvenile rheumatoid arthritis.

Keywords - Hip; Arthritis, Juvenile Rheumatoid; Orthopedic Procedures

1 - Professor Associado da Faculdade de Medicina da Universidade de São Paulo; Chefe da Disciplina de Ortopedia Pediátrica do Departamento de Ortopedia São Paulo, SP.

2 - Professor Colaborador da Faculdade de Medicina da Universidade de São Paulo; Médico Assistente do Instituto de Ortopedia e Traumatologia do HC/FMUSP São Paulo, SP.

3 - Médicos Assistentes do Instituto de Ortopedia e Traumatologia do HC/FMUSP - São Paulo, SP.

4 - Professora Livre-Docente, Faculdade de Medicina, Universidade de São Paulo (FMUSP) - São Paulo, SP.

Trabalho realizado no Instituto de Ortopedia e Traumatologia do Hospital das Clínicas da Faculdade de Medicina da Universidade de São Paulo Correspondência: Rua Dr. Ovídio Pires de Campos, 333 - $3^{\circ}$ andar - Cerqueira Cesar - 05403-010 - São Paulo, SP. E-mail: robertoguarniero@gmail.com 


\section{INTRODUÇÃO}

Os objetivos do tratamento ortopédico na artrite reumatoide juvenil (ARJ) incluem a prevenção e a correção das deformidades articulares e a preservação do grau normal de movimentação nas diferentes articulações; isto pode ser efetuado e obtido, por exemplo, pela tração ou pela reabilitação fisioterápica. A movimentação, na amplitude próxima do normal, é fundamental para o processo normal de nutrição da cartilagem articular. Então, a recuperação dos movimentos de uma articulação rígida e com alterações degenerativas, tornará a mesma estável e indolor.

A artrodiastase é uma forma de tração articular, efetuada por um fixador externo, com a finalidade de restaurar os movimentos articulares porventura bloqueados. A mobilização de uma articulação ao mesmo tempo em que as suas superfícies articulares são mantidas afastadas, em tração, por um fixador externo, foi descrita em 1975 por Volkov e Oganesian $^{(1)}$, utilizando um fixador externo de tipo circular. Os resultados da utilização clínica da técnica de artrodiastase, na articulação coxofemoral, foram publicados por Aldegheri et $a l^{(2)} \mathrm{e}$, mais recentemente, por Cañadell et $a l^{(3)}$; entretanto, nestes artigos não são relatados casos clínicos de artrite reumatoide juvenil.

Neste trabalho prospectivo, apresentamos os resultados preliminares desta modalidade de tratamento em pacientes portadores de ARJ e com comprometimento da articulação coxofemoral, acompanhados no Departamento de Ortopedia e Traumatologia e no Instituto da Criança da Faculdade de Medicina da Universidade de São Paulo, no período de 1993 a 2008.

\section{MATERIAL E MÉTODO}

No período de 1993 a 2008, 12 pacientes (seis meninos e seis meninas), com idades variando entre oito e 18 anos (média de 10,5 anos), com diagnóstico de ARJ e comprometimento da articulação coxofemoral, foram submetidos ao tratamento com a técnica de artrodiastase, no Departamento de Ortopedia e Traumatologia e no Instituto de Criança da Faculdade de Medicina da Universidade de São Paulo.

Foi utilizado um fixador externo monolateral, tipo "Orthofix", com um mecanismo que permite os movimentos de flexão e de extensão no quadril, com dois pinos tipo Schanz inseridos no osso ilíaco e dois outros na região proximal da diáfise femoral. $\mathrm{O}$ fixador externo foi mantido por um período que variou de 78 a 90 dias, com média de 86 dias, realizando o afastamento entre as superfícies articulares da cavidade acetabular e da cabeça femoral.

A tração efetuada pelo fixador era iniciada no ato operatório $(0,5$ a $1,0 \mathrm{~cm}$ de afastamento) e prolongavase por, aproximadamente, 10 a 12 dias consecutivos no pós-operatório. A seguir, o fixador externo era bloqueado quanto ao alongamento, mas permitindo a movimentação de flexoextensão na articulação coxofemoral.

O controle radiográfico foi realizado na projeção posteroanterior da articulação do quadril durante o ato operatório e, a seguir, semanalmente, durante o período de tração e a cada quatro semanas, quando terminado este período de tração.

$\mathrm{Na}$ avaliação clínica dos resultados, incluímos a graduação da dor, em uma escala de 1 a 10 conforme a intensidade e o grau de movimentação articular, com medidas e avaliações pré e pós-operatórias ${ }^{(4,5)}$. No pós-operatório, os pacientes foram examinados a cada quatro semanas.

$\mathrm{O}$ período de acompanhamento variou de 12 a 15 anos, com média de 13 anos.

\section{RESULTADOS}

Na Tabela 1, são mostrados os resultados em relação à escala da dor na articulação do quadril no pré e pósoperatório para a faixa etária pediátrica. Somente em dois pacientes não ocorreu melhora da dor no quadril. $\mathrm{O}$ valor médio da escala foi de nove (9) antes da operação e de quatro (4) no período pós-operatório.

Tabela 1 - Valores da escala da dor, pré e pós-operatório.

\begin{tabular}{c|c|c}
\hline Paciente & Pré-operatório & Pós-operatório \\
\hline 1 & 9 & 2 \\
\hline 2 & 9 & 7 \\
\hline 3 & 9 & 1 \\
\hline 4 & 9 & 2 \\
\hline 5 & 9 & 9 \\
\hline 6 & 9 & 8 \\
\hline 7 & 9 & 3 \\
\hline 8 & 9 & 5 \\
\hline 9 & 10 & 2 \\
\hline 10 & 10 & 3 \\
\hline 11 & 8 & 3 \\
\hline 12 & 8 & 2 \\
\hline
\end{tabular}

Fonte: IOT - Hospital das Clínicas FMUSP

$\mathrm{O}$ arco de movimento da articulação coxofemoral aumentou em todos os pacientes, com exceção de dois. Na Tabela 2, mostramos o resultado médio desta 
Tabela 2 - Valor médio (), pré e pós-operatório, do arco de movimento do quadril operado.

\begin{tabular}{c|c|c|c|c|c|c}
\hline Valor & Flexão & Extensão & Abdução & Adução & Rot. ext. & Rot. int. \\
\hline Média pré & 35,5 & 9,5 & 14 & 4,5 & 10 & 7 \\
\hline Média pós & 53,5 & 12,5 & 23 & 9,5 & 15,5 & 12,5 \\
\hline
\end{tabular}

Fonte: IOT - Hospital das Clínicas FMUSP Rot. ext. = Rotação externa Rot. int. = Rotação interna

avaliação clínica, incluindo os movimentos do quadril: flexão, extensão, abdução, adução, rotação externa e rotação interna. $\mathrm{Na}$ avaliação radiográfica evidenciamos um aumento no espaço articular de $2 \mathrm{~mm}$, em média, e que se mantém no pós-operatório.

Não foram observadas complicações com a utilização da técnica. Apenas verificamos a soltura dos pinos de Schanz da região do osso ilíaco em dois pacientes, decorridos 35 e 42 dias após a sua colocação. Os pinos foram substituídos nos dois pacientes. Não ocorreram infecções.

Em dois pacientes, a técnica produziu resultado satisfatório. Estes dois pacientes eram os que tinham idade mais elevada quando do procedimento operatório: $18 \mathrm{e}$ 17 anos, respectivamente. Em ambos, já foi realizada artroplastia total do quadril comprometido.

Nas Figuras 1, 2 e 3, mostramos aspectos do procedimento técnico.

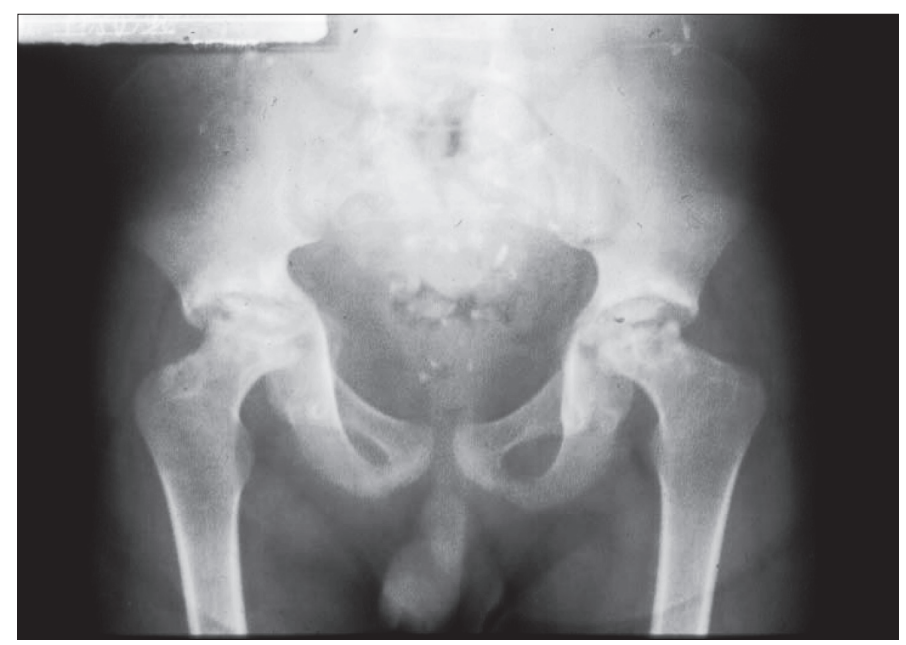

Figura 1 - Radiografia de paciente com 10 anos de idade, masculino e comprometimento bilateral.

\section{DISCUSSÃO}

Concordamos com Kiss ${ }^{(6)}$, Brewer et $a l^{(7)}$, Harris e Baum $^{(8)}$ e Jacobsen et $a l^{(9)}$, quando afirmam que, na artrite reumatoide juvenil (ARJ), o comprometimento das articulações coxofemorais e dos joelhos, por exemplo, revestem-se habitualmente de maior gravidade funcional, quando comparado ao envolvimento de outras articulações. Para Brewer et $a l^{(7)}$, o quadril é a articulação mais importante em crianças com ARJ,

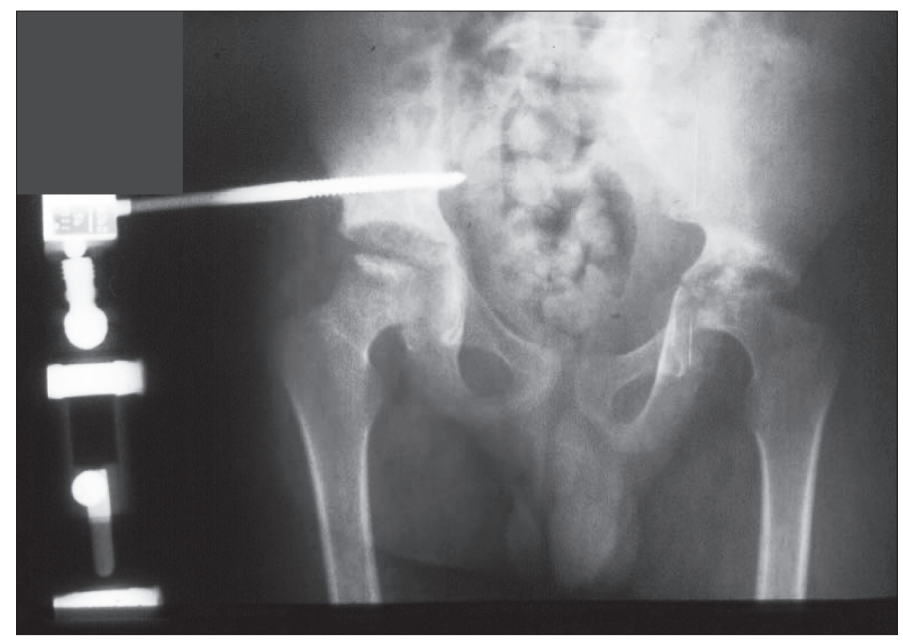

Figura 2 - Radiografia mostrando evolução do tratamento.
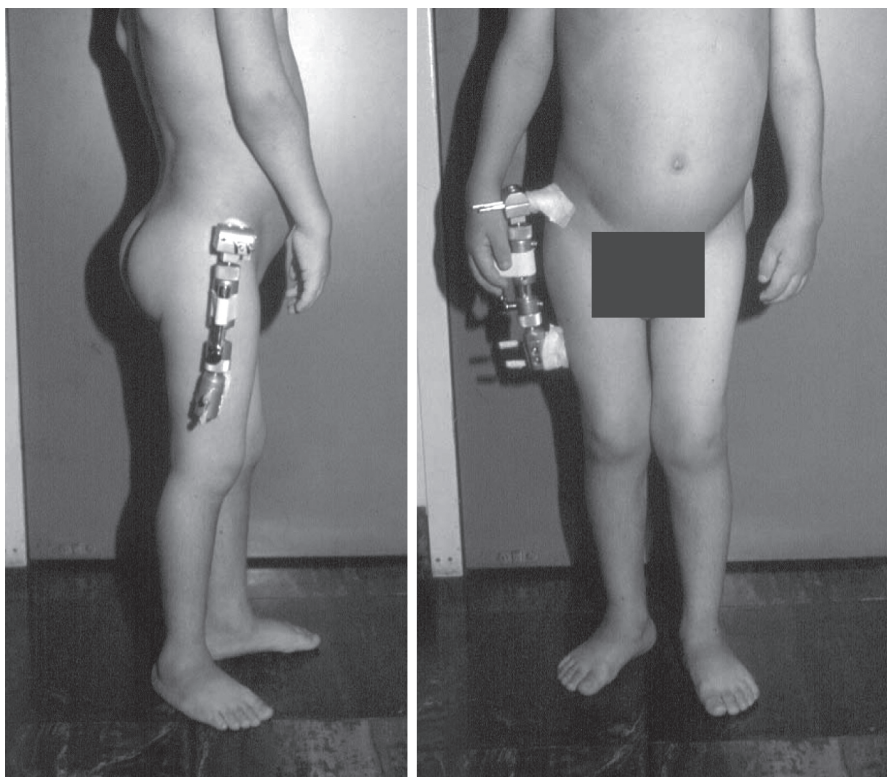

Figura 3 - Aspecto clínico. Pós-operatório imediato.

sendo o seu comprometimento a primeira causa de incapacitação grave. Ainda, como refere $\mathrm{Kiss}^{(6)}$, o comprometimento da articulação coxofemoral é bastante comum nos pacientes que procuram a nossa instituição para o tratamento; esta incidência de comprometimento coxofemoral é de $70 \%$, e é um determinante importante de mau prognóstico funcional.

As cirurgias ortopédicas são sistematicamente indicadas para a melhora da função e/ou da dor persistente, incapacitante, que os pacientes apresentam. Particular- 
mente, em relação à articulação do quadril, dispomos de poucas alternativas para o tratamento ortopédico operatório. Bons resultados, evidentemente, poderão ser obtidos pela artrodese do quadril ou, ainda, pela artroplastia. Entretanto, ambos os procedimentos estão indicados para o paciente em uma faixa etária maior, na faixa de indicação de um procedimento operatório do tipo "de salvação". Pelos resultados preliminares obtidos nesta série estudada, acreditamos que a artrodiastase pode ser a indicação de escolha na ARJ para os pacientes com acometimento da coxofemoral e na faixa etária de cinco a 16 anos.

Como refere Ansell ${ }^{(10)}$, os prejuízos em relação ao crescimento são acentuados, e as crianças mais jovens apresentam prejuízos funcionais mais acentuados pela agressão articular em articulações incompletamente desenvolvidas. Concordando uma vez mais com Kiss ${ }^{(6)}$, este problema é muito importante, em especial nas articulações coxofemorais, em que a marcha é considerada fundamental para o desenvolvimento anatômico e funcional adequado do quadril. Jacobsen et al ${ }^{(9)}$ referem que o envolvimento da articulação coxofemoral, em crianças com a forma sistêmica e com idades inferiores a seis anos, associa-se a pior prognóstico e a alterações radiológicas mais frequentes. Para estes casos também está indicada a técnica da artrodiastase, pois, como verificamos em nossos resultados, há uma evidente melhora da imagem radiográfica da articulação após a retirada do fixador externo, e que tem se mantido inalterada, até o presente acompanhamento dos pacientes.

Concordamos com Cañadell et al $l^{(3)}$ que afirmam ser fundamental a manutenção de um programa de reabilitação intenso após a retirada do fixador externo, com ênfase especial para a conservação do grau de movimentação de flexoextensão do quadril, obtido pela utilização da artrodiastase.

O fixador externo será mantido até que as radiografias revelem a mesma distância de afastamento entre as superfícies articulares por pelo menos um mês.

\section{CONCLUSÃO}

Como conclusão, podemos afirmar que o procedimento de artrodiastase do quadril está bem indicado para a recuperação da mobilidade em uma articulação coxofemoral comprometida e rígida. Um fato muito importante é que a dor é aliviada de maneira substancial nos pacientes portadores de ARJ tratados com a técnica, até a presente data.

\section{REFERÊNCIAS}

1. Volkov MV, Oganesian OV. Restoration of function in the knee and elbow with a hinge-distractor apparatus. J Bone Joint Surg Am. 1975;57(5):591-600.

2. Aldegheri R, Agostini S, Renzi Brivio L. Artrodiatasi d'anca (esperienza clinica). Chir Organi Mov. 1982;68(4/5):527-31.

3. Cañadell J, Gonzales F, Barrios RH, Amillo S. Arthrodiastasis for stiff hips in young patients. Int Orthop. 1993;17(4):254-8.

4. Castellanos AL, Silva CA. Dor musculoesquelética idiopática e recorrente na faixa etária pediátrica. In: Silva CA. Doenças reumáticas na criança e no adolescente. Barueri, SP: Manole; 2008. p. 65-78.

5. Castro WH, Jerosch J. Exame e diagnóstico dos distúrbios musculoesqueléticos. Porto Alegre: Artmed; 2005. p. 190.
6. Kiss MHB. Estudo sobre a determinação de um índice prognóstico em crianças portadoras de Artrite Reumatóide Juvenil (ARJ) [tese - livre docência]. São Paulo: Faculdade de Medicina da Universidade de São Paulo; 1994.

7. Brewer EJ, Giannini EH, Person, DA, editors. Orthopedic management in juvenile chronic arthritis. 2nd ed. Philadelphia: Saunders; 1982. p. 229-88.

8. Harris CM, Baum J. Involvement of the hip in juvenile rheumatoid arthritis. A longitudinal study. J Bone Joint Surg Br. 1988;70(6):821-33.

9. Jacobsen FS, Crawford $\mathrm{AH}$, Broste $\mathrm{S}$. Hip involvement in juvenile rheumatoid arthritis. J Pediatr Orthop. 1992;12(1):45-53.

10. Ansell BM. Juvenile chronic arthritis. Scand J Rheumatol. 1987;66(Suppl): 47-50. 